\title{
A CLASS OF PLANAR QUADRATIC VECTOR FIELDS WITH A LIMIT CYCLE SURROUNDED BY A SADDLE LOOP
}

\author{
RODRIGO BAMÓN
}

\begin{abstract}
Examples of planar quadratic vector fields with a limit cycle surrounded
\end{abstract} by a saddle loop are given by means of deformations of Hamiltonian vector fields.

1. Statement of the main result. It is known that if a planar quadratic vector field $X$ has infinitely many limit cycles, then they necessarily accumulate in a separatrix cycle of the Poincare compactification of $X$. In $[\mathbf{C h S h}]$ it is proved that a bounded separatrix cycle surrounds at most finitely many limit cycles. However, no example of at least one limit cycle surrounded by a separatrix cycle is known [ChT, ChSh]. In this note we give examples of this situation by using small deformations of Hamiltonian vector fields.

Let $\mathfrak{X}^{2}$ denote the vector space of planar quadratic vector fields.

THEOREM. There exists a codimension one analytic submanifold $S$ of $\mathfrak{X}^{2}$ such that every vector field in $S$ has a limit cycle surrounded by a saddle loop.

2. Some preliminary lemmas. Let

$$
X(x, y)=\left(2 y^{2}-2 y\right) \frac{\partial}{\partial x}-\left(x+x^{2}\right) \frac{\partial}{\partial y}
$$

be the planar Hamiltonian quadratic vector field with potential function

$$
H(x, y)=\frac{1}{3} x^{3}+\frac{1}{2} x^{2}+\frac{2}{3} y^{3}-y^{2} .
$$

The level curves of $H$ are given in Figure 1(a). We see that $X$ has a saddle at $p_{0}=(0,0)$, a center at $p_{1}=(-1,0)$ and a saddle loop $\gamma$ at $p_{0}$. The saddle loop $\gamma$ is contained in the zero level curve of $H$. Let $A_{0}$ be the region bounded by $\gamma \cup\left\{p_{0}\right\}$.

LEMma 1. The reflection of $\gamma \cap\{y<0\}$ with respect to the line $y=0$ is contained in $A_{0}$.

Proof. Let $(x, y) \in \gamma \cap\{y<0\}$. Then $H(x, y)=0$ and $H(x,-y)=H(x, y)$ $-\frac{4}{3} y^{3}=-\frac{4}{3} y^{3}>0$.

That is, the reflection of $(x, y)$ lies in a positive level curve of $H$. Noticing that the shadowed region in Figure 1(b) is the one where $H$ is positive and the reflections of all points in $\gamma \cap\{y<0\}$ are in this region, we necessarily have that the reflection of this curve is contained in $A_{0}$.

Received by the editors September 21, 1982.

1980 Mathematics Subject Classification. Primary 58F21; Secondary 34C05.

Key words and phrases. Limit cycle, quadratic systems, saddle loop, Hamiltonian.

(C)1983 American Mathematical Society $0002-9939 / 83 / 0000-1317 / \$ 02.25$ 


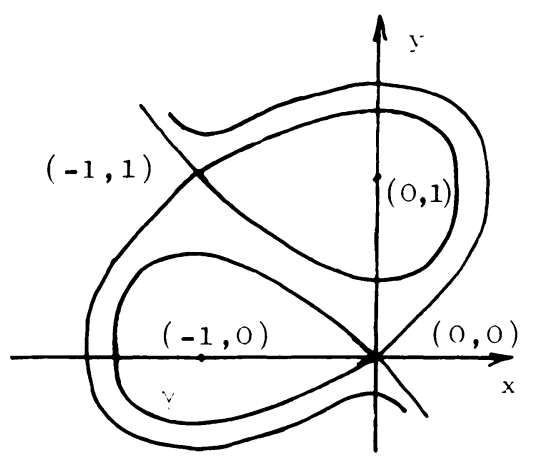

Figure 1(a)

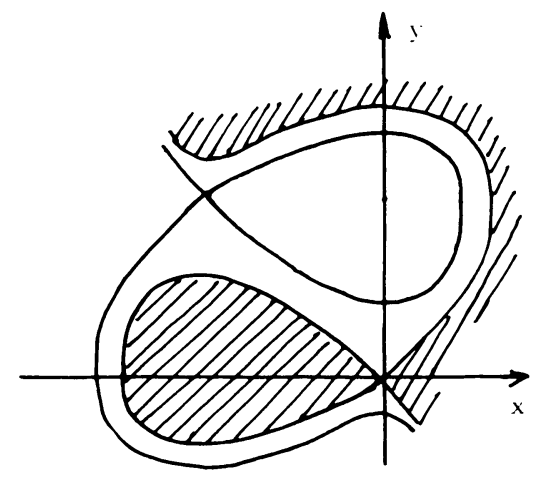

Figure 1(b)

Let $\Sigma=\{(x, 0) \mid-2<x<-5 / 4\}$ be a transversal section of $X$ that intersects $\gamma$ at the point $q=(-3 / 2,0)$.

Let $N$ be a neighbourhood of $X$ in $\mathfrak{X}^{2}$ such that:

(i) There exists $p_{0}: N \rightarrow \mathbf{R}^{2}$ analytic with $p_{0}(X)=p_{0}$ and $Y\left(p_{0}(Y)\right)=0$ for all $Y \in N$.

(ii) There exists $p_{1}: N \rightarrow \mathbf{R}^{2}$ analytic with $p_{1}(X)=p_{1}$ and $Y\left(p_{1}(Y)\right)=0$ for all $Y \in N$.

(iii) Each $Y \in N$ is transversal to $\Sigma$.

(iv) The separatrices $W^{u}(Y)$ and $W^{s}(Y)$ of the saddle $p_{0}(Y)$ intersect $\Sigma$ at the points $\left(q^{u}(Y), 0\right)$ and $\left(q^{\prime}(Y), 0\right)$, respectively, as shown in Figure 2.

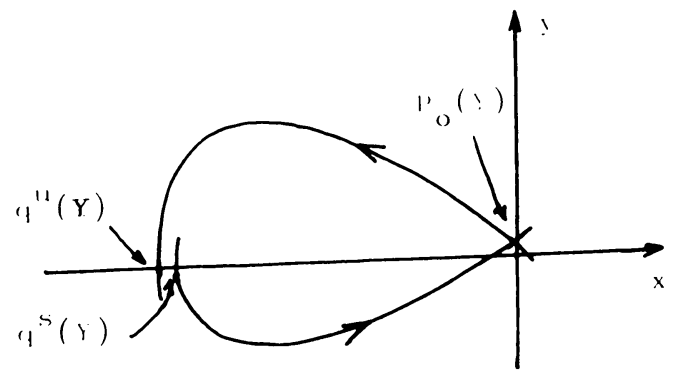

FI(jURI: 2

We have the following facts:

(1) $F: N \rightarrow \mathbf{R}$, defined by $F(Y) \cdots q^{\prime \prime}(Y)-q^{\prime}(Y)$, is analytic with zero as a regular value (see $[\mathbf{S}])$. Also)

$$
T_{X} F^{\prime}(0)=\left\{Z \in \mathfrak{X}^{2} \mid \iint_{\Lambda_{0}} \operatorname{div} Z d x d y=0\right\} .
$$

Following Sotomayor [S], we will prove the last assertions. 
In coordinates suppose the following situation for $W^{s}(X)$ :

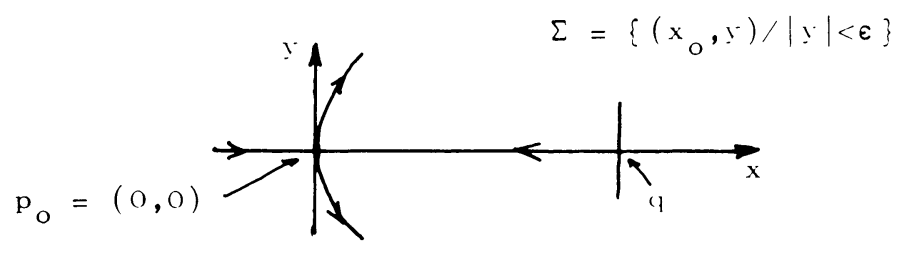

We compute

$$
\frac{\partial q^{s}}{\partial z}(Y)=\lim _{h \rightarrow 0} \frac{q^{s}(Y+h Z)-q^{s}(Y)}{h} .
$$

Let $\gamma(h, t), t \geqslant 0$, be the solution of $Y+h Z$ such that $\gamma(h, 0)=q^{s}(Y+h Z)$. Then $v(t)=\partial \gamma(0, t) / \partial h$ satisfies

$$
\dot{v}_{1}=\frac{\partial Y_{1}}{\partial x} v_{1}+\frac{\partial Y_{1}}{\partial y} v_{2}+Z_{1}, \quad \dot{v}_{2}=\frac{\partial Y_{2}}{\partial x} v_{1}+\frac{\partial Y_{2}}{\partial y} v_{2}+Z_{2}
$$

The component $n(t)$ of $\left(v_{1}(t), v_{2}(t)\right)$ in the normal direction to $Y(t)=Y(\gamma(0, t))$ satisfies

$$
\dot{n}=(\operatorname{div} Y-\mid \overbrace{Y|/| Y \mid}^{e} n+(1 /|Y|) \operatorname{det}(Y, Z) \text {. }
$$

So

$$
\begin{aligned}
n(t)= & \frac{|Y(0)|}{|Y(t)|} \exp \left(\int_{0}^{t} \operatorname{div} Y(\tau) d \tau\right) \\
& \times\left[n(0)+\frac{1}{|Y(0)|} \int_{0}^{t} \exp \left(-\int_{0}^{\tau} \operatorname{div} Y(u) d u\right) \operatorname{det}(Y, Z) d \tau\right]
\end{aligned}
$$

and then

$$
\begin{aligned}
n(0)= & n(t) \frac{|Y(t)|}{|Y(0)|} \exp \left(-\int_{0}^{t} \operatorname{div} Y(\tau) d \tau\right) \\
& -\frac{1}{|Y(0)|} \int_{0}^{t} \exp \left(-\int_{0}^{\tau} \operatorname{div} Y(u) d u\right) \operatorname{det}(Y, Z) d \tau
\end{aligned}
$$

Since the integral

$$
\int_{0}^{\infty}|Y(t)| \exp \left(-\int_{0}^{t} \operatorname{div} Y(\tau) d \tau\right) d t
$$

converges [CL, p. 331] we have

$$
\lim _{t \rightarrow \infty}|Y(t)| \exp \left(-\int_{0}^{t} \operatorname{div} Y(\tau) d \tau\right)=0 .
$$

Therefore

$$
n(0)=-\frac{1}{|Y(0)|} \int_{0}^{\infty} \exp \left(-\int_{0}^{\tau} \operatorname{div} Y(u) d u\right) \operatorname{det}(Y, Z) d \tau
$$


But $\partial q^{s}(Y) / \partial Z=\partial \gamma(0,0) / \partial h=n(0) / \cos \alpha, \quad \alpha$ being the angle determined by $Y\left(q^{s}(Y)\right)$ and $X(q)$.

So

$$
\frac{\partial q^{s}}{\partial Z}(Y)=-\frac{|X(q)|}{\langle X(q), Y(0)\rangle} \int_{0}^{\infty} \exp \left(-\int_{0}^{\tau} \operatorname{div} Y(u) d u\right) \operatorname{det}(Y, Z) d \tau .
$$

Similarly,

$$
\frac{\partial q^{u}}{\partial Z}(Y)=-\frac{|X(q)|}{\langle X(q), Y(0)\rangle} \int_{0}^{-\infty} \exp \left(-\int_{0}^{\tau} \operatorname{div} Y(u) d u\right) \operatorname{det}(Y, Z) d \tau .
$$

Since $X$ is Hamiltonian we obtain

$$
\begin{aligned}
\frac{\partial F}{\partial Z}(X) & =\frac{\partial q^{u}}{\partial Z}(X)-\frac{\partial q^{s}}{\partial Z}(X)=\frac{1}{|X(q)|} \int_{-\infty}^{\infty} \operatorname{det}(X, Z) d \tau \\
& =-\frac{1}{|X(q)|} \iint_{A_{0}} \operatorname{div} Z d x d y
\end{aligned}
$$

where the last equality is Green's formula.

It is an easy fact to prove that there exists $Z \in \mathfrak{X}^{2}$ such that $\iint_{A_{0}} \operatorname{div} Z d x d y \neq 0$ (see proof of Lemma 2 below). That is, zero is a regular value of $F$ and

$$
T_{X} F^{-1}(0)=\left\{Z \in \mathfrak{X}^{2} \mid \iint_{A_{0}} \operatorname{div} Z(x, y) d x d y=0\right\} .
$$

(2) Each $D_{i}: N \rightarrow \mathbf{R}, i=0,1$, defined by $D_{i}(Y)=\operatorname{div} Y\left(p_{i}(Y)\right)$, is analytic with zero as a regular value. By a direct calculation,

$$
T_{X} D_{i}^{-1}(0)=\left\{Z \in \mathfrak{X}^{2} \mid \operatorname{div} Z\left(p_{i}\right)=0\right\} .
$$

Lemma 2. There exists $Z \in \mathfrak{X}^{2}$ such that $\iint_{A_{0}} \operatorname{div} Z d x d y=0, \operatorname{div} Z\left(p_{0}\right) \neq 0$ and $\operatorname{div} Z\left(p_{1}\right) \neq 0$.

Proof. Let

$$
Z_{\varepsilon}(x, y)=-\varepsilon x \frac{\partial}{\partial x}+\frac{y^{2}}{2} \frac{\partial}{\partial y} .
$$

Then $\operatorname{div} Z_{\varepsilon}(x, y)=-\varepsilon+y$ and, by Lemma 1 ,

$$
\iint_{A_{0}} \operatorname{div} Z_{0}(x, y) d x d y=\iint_{A_{0}} y d x d y>0
$$

Also

$$
\iint_{A_{0}} \operatorname{div} Z_{1}(x, y) d x d y=\iint_{A_{0}}(y-1) d x d y<0
$$

because $y-1<0$ for all $(x, y) \in A_{0}$. By continuity, there exists $\left.\varepsilon_{0} \in\right] 0,1[$ such that

$$
\iint_{A_{0}} \operatorname{div} Z_{\varepsilon_{0}}(x, y) d x d y=0 .
$$

For this $\varepsilon_{0}, \operatorname{div} Z_{\varepsilon_{0}}\left(p_{i}\right)=-\varepsilon_{0}<0, i=0,1$. 
Lemma 3. The vector spaces $T_{X} F^{-1}(0), T_{X} D_{0}^{-1}(0)$ and $T_{X} D_{1}^{-1}(0)$ are in general position.

Proof. By Lemma 2, $T_{X} F^{-1}(0) \pitchfork T_{X} D_{i}^{-1}(0), i=0,1$. Now $Z(x, y)=x^{2}(\partial / \partial x)$ belongs to $T_{X} D_{0}^{-1}(0)$, but not to $T_{X} D_{1}^{-1}(0)$. That is, $T_{X} D_{0}^{-1}(0) \pi T_{X} D_{1}^{-1}(0)$.

Now we prove that there exists a vector field $Z \in \mathfrak{X}^{2}$ so that $Z \in T_{X} D_{0}^{-1}(0) \cap$ $T_{X} F^{-1}(0)$ and $Z \notin T_{X} D_{1}^{-1}(0)$. This will complete the proof.

Let $Z_{\lambda}(x, y)=\frac{1}{2}\left(\lambda x^{2}(\partial / \partial x)+y^{2}(\partial / \partial y)\right)$.

Then $\operatorname{div} Z_{\lambda}(x, y)=\lambda x+y$ and, by Lemma 1 .

$$
\iint_{A_{0}} \operatorname{div} Z_{0}(x, y) d x d y=\iint_{A_{0}} y d x d y>0 .
$$

For $\lambda$ big enough (in fact $\lambda \geqslant \frac{1}{2} \sqrt{2}$ ) we have $\operatorname{div} Z_{\lambda}(x, y)<0$ for all $(x, y) \in A_{0}$. So

$$
\iint_{A_{0}} \operatorname{div} Z_{\lambda}(x, y) d x d y<0
$$

By continuity there exists $\lambda_{0}>0$ (less than $\frac{1}{2} \sqrt{2}$ ) such that $\iint_{A_{0}} \operatorname{div} Z_{\lambda_{0}}(x, y) d x d y=$ 0 . Since $\operatorname{div} Z_{\lambda_{0}}\left(p_{0}\right)=0$ and $\operatorname{div} Z_{\lambda_{0}}\left(p_{1}\right)=-\lambda_{0} \neq 0$, the vector field $Z_{\lambda_{0}}$ belongs to $T_{X} D_{0}^{-1}(0) \cap T_{X} F^{-1}(0)$, but not to $T_{X} D_{1}^{-1}(0)$.

3. Proof of the main result. By Lemma $3, F^{-1}(0), D_{0}^{-1}(0)$ and $D_{1}^{-1}(0)$ are in general position at $X$. Choosing the neighbourhood $N$ small enough we can suppose that they are in general position at each common vector field $Y \in F^{-1}(0) \cap D_{0}^{-1} \cap D_{1}^{-1}(0)$ and each $F^{-1}(0), D_{0}^{-1}(0)$ or $D_{1}^{-1}(0)$ separates $N$ into the two natural regions.

Each $Y \in F^{-1}(0)$ with $D_{0}(Y) D_{1}(Y)>0$ has a saddle loop at $p_{0}(Y)$ and a hyperbolic focus at $p_{1}(Y)$, both stable or unstable depending on the sign of $D_{0}(Y)$. That is, one of the following situations happens:
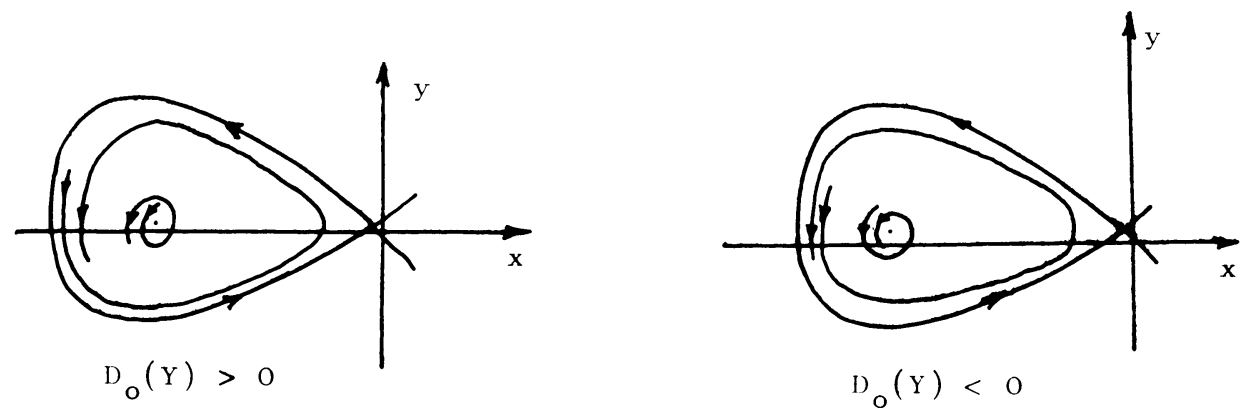

In each case $Y$ must have a limit cycle. Then $S=\left\{Y \in F^{-1}(0) \mid D_{0}(Y) D_{1}(Y)>0\right\}$ is the required codimension one analytic submanifold of $\mathfrak{X}^{2}$.

4. Acknowledgement. I wish to thank Professor Jorge Sotomayor for his helpful advice in the preparation of this paper. I also wish to thank Professor Douglas Shafter for his uselful comments on the original version. 


\section{REFERENCES}

[CL] E. Coddington and N. Levinson, Theory of ordinary differential equations, McGraw-Hill, New York, 1955.

[ChSh] C. Chicone and D. Shafer, Separatrix and limit cycles of quadratic systems and Dulac's theorem, Trans. Amer. Math. Soc. (to appear).

[ChT] C. Chicone and Tian Jinghiang, On general properties of quadratic systems, Amer. Math. Monthly 89 (1982), no. 3.

[S] J. Sotomayor, Estabilidade estrutural de primeira ordem e variedades de Banach, Thesis, IMPA, Brazil, 1964.

Instituto de Matemática Pura e Aplicada, CEP 22460, Rio de Janeiro, RJ-Brazil

Universidad de Chile, Santiago, Chile 